\title{
Nonequilibrium mesoscopic conductance fluctuations
}

\author{
T. Ludwig, ${ }^{1}$ Ya. M. Blanter, ${ }^{2}$ and A. D. Mirlin ${ }^{1,3, *}$ \\ ${ }^{1}$ Institut für Nanotechnologie, Forschungszentrum Karlsruhe, 76021 Karlsruhe, Germany \\ ${ }^{2}$ Kavli Institute of Nanoscience, Delft University of Technology, Lorentzweg 1, 2628 CJ Delft, The Netherlands \\ ${ }^{3}$ Institut für Theorie der Kondensierten Materie, Universität Karlsruhe, 76128 Karlsruhe, Germany \\ (Received 2 June 2004; revised manuscript received 30 August 2004; published 13 December 2004)
}

\begin{abstract}
We investigate the amplitude of mesoscopic fluctuations of the differential conductance of a metallic wire at arbitrary bias voltage $V$. For noninteracting electrons, the variance $\left\langle\delta g^{2}\right\rangle$ increases with $V$. The asymptotic large- $V$ behavior is $\left\langle\delta g^{2}\right\rangle \sim V / V_{c}$ (where $e V_{c}=D / L^{2}$ is the Thouless energy), in agreement with the earlier prediction by Larkin and Khmelnitskii. We find, however, that this asymptotics has a very small numerical prefactor and sets in at very large $V / V_{c}$ only, which strongly complicates its experimental observation. This high-voltage behavior is preceded by a crossover regime, $V / V_{c} \lesssim 30$, where the conductance variance increases by a factor $\sim 3$ as compared to its value in the regime of universal conductance fluctuations (i.e., at $V \rightarrow 0$ ). We further analyze the effect of dephasing due to the electron-electron scattering on $\left\langle\delta g^{2}\right\rangle$ at high voltages. With the Coulomb interaction taken into account, the amplitude of conductance fluctuations becomes a nonmonotonic function of $V$. Specifically, $\left\langle\delta g^{2}\right\rangle$ drops as $1 / V$ for voltages $V \gg g V_{c}$, where $g$ is the dimensionless conductance. In this regime, the conductance fluctuations are dominated by quantum-coherent regions of the wire adjacent to the reservoirs.
\end{abstract}

DOI: 10.1103/PhysRevB.70.235315

\section{INTRODUCTION}

Universal conductance fluctuations ${ }^{1-3}$ (UCF) represent one of the most famous manifestations of quantum-coherent electron transport in mesoscopic conductors. Due to the quantum coherence of the electron motion over the entire sample, the variance $\left\langle\delta g^{2}\right\rangle$ of the dimensionless (measured in units of $e^{2} / h$ ) conductance $g$ is of order unity, with no dependence on the system size. The numerical coefficient depends on the spatial dimensionality and on global symmetries of the Hamiltonian. In particular, for a quasi-onedimensional system (a wire) and for preserved time-reversal and spin-rotation symmetries one has $\left\langle\delta g^{2}\right\rangle=8 / 15$.

In a pioneering paper ${ }^{4}$ Larkin and Khmel'nitskii (LK) predicted an enhancement of mesoscopic fluctuations of the differential conductance $g=d I / d V$ at high bias voltages $V$. Specifically, they found that for $V \gg V_{c}$, where $e V_{c}=D / L^{2}$ is the Thouless energy, $D$ the diffusion constant, and $L$ the system size, the variance of the conductance increases linearly with $V,\left\langle\delta g^{2}\right\rangle \sim V / V_{c}$. This result implies, in particular, that regions of negative differential resistance should appear in the $I V$ characteristics of a mesoscopic sample at sufficiently high voltages, $V / V_{c} \gtrsim g^{2}$.

While early measurements ${ }^{5-7}$ did not confirm the predicted enhancement of the conductance fluctuations by the bias voltage, more recent experiments ${ }^{8-11}$ did observe an increase of $\left\langle\delta g^{2}\right\rangle$ with $V$. This increase was interpreted as the linear behavior $\left\langle\delta g^{2}\right\rangle \sim V / V_{c}$ predicted by LK.

Inelastic scattering processes lead to dephasing, thus suppressing interference phenomena like mesoscopic conductance fluctuations. Indeed, it was found in all experiments that at sufficiently high voltages the amplitude of conductance fluctuations starts to decrease with $V$. However, no quantitative theory of the effect of Coulomb interaction on the conductance fluctuations has been developed. $\mathrm{LK}^{4}$ characterized the effect of inelastic scattering by a phenomeno-
PACS number(s): 73.23.-b, 73.63.Nm, 72.20.Ht

logical inelastic out-scattering time $\tau_{\text {in }}$ and the corresponding length $L_{\text {in }}=\sqrt{D \tau_{\text {in }}}$. They obtained a suppression factor of $\left\langle\delta g^{2}\right\rangle$ of the form $\sim\left(L_{\text {in }} / L\right)^{7}$ for the case $L_{\text {in }} \ll L$. No microscopic theory for $L_{\text {in }}$ (which would produce, in particular, its explicit dependence on $V$ ) was presented in Ref. 4. The authors of Refs. 10 and 11 identified the $V$-dependent dephasing length $L_{\phi}(V)$ as a relevant length scale induced by the inelastic scattering. They further argued that for the case of Coulomb interaction $L_{\phi}(V)$ can be obtained from the known result $^{12,13}$ for $L_{\phi}(T)$ by a replacement $T \rightarrow V$. They estimated the suppression factor of $\left\langle\delta g^{2}\right\rangle$ by the inelastic processes as $\left[L_{\phi}(V) / L\right]^{4}$, at variance with LK. It was found experimentally in Ref. 10 that $\left\langle\delta g^{2}\right\rangle$ decreases as $V^{-\gamma}$ at large $V$, with $\gamma=1.28 \pm 0.12$. The authors argued that the electron-electron scattering is not sufficient to convert the LK enhancement mechanism into such a fall-off, and ascribed this behavior to the electron-phonon scattering.

The purpose of this paper is to develop a systematic theory of the voltage dependence of conductance fluctuations of mesoscopic wires. In Sec. II we calculate explicitly the dependence of $\left\langle\delta g^{2}\right\rangle$ on $V / V_{c}$ for not too high voltages $V \ll g V_{c}$, when (as we will see in Sec. IV) the electronelectron scattering processes do not play any role. While we confirm the LK asymptotic result $\left\langle\delta g^{2}\right\rangle \sim V / V_{c}$ at $V \gg V_{c}$, we find that the corresponding contribution has a very small numerical prefactor. As a result, the LK asymptotic behavior sets in at very large $V / V_{c}$ only and can hardly be detected experimentally. This high-voltage behavior is preceded by a crossover regime, $V / V_{c} \lesssim 30$, where the conductance variance increases by a factor $\sim 3$ as compared to its value in the regime of universal conductance fluctuations (i.e., at $V \rightarrow 0$ ). In contrast to the weak increase of the conductance fluctuations in the LK regime, this strong enhancement of $\left\langle\delta g^{2}\right\rangle$ in the crossover regime can easily be verified experimentally. In particular, we believe that it is this behavior that has 
been observed in the most recent and detailed experimental study. ${ }^{10,11}$

In Sec. III we consider the effect of finite temperature, $T \gtrsim e V_{c}$. We show that the temperature does not affect the conductance fluctuations at high voltages $e V \gg T$ (up to a constant offset) but strongly suppresses them at lower voltages $e V \ll T$.

In Sec. IV we analyze the effect of dephasing due to the electron-electron interaction on $\left\langle\delta g^{2}\right\rangle$ at high voltages. We show that the Coulomb interaction makes the amplitude of conductance fluctuations a nonmonotonic function of $V$. Specifically, we find that $\left\langle\delta g^{2}\right\rangle$ drops as $1 / V$ for voltages $V \gg g V_{c}$, where $g$ is the dimensionless conductance. Our results demonstrate that in the nonequilibrium situation the sample cannot be characterized by a single dephasing length, since the latter is position-dependent and strongly increases near the contacts with the reservoirs. Furthermore, we show that the conductance fluctuations are dominated by quantumcoherent segments of the wire adjacent to the reservoirs. According to our results, the maximal rms conductance fluctuations $\sqrt{\left\langle\delta g^{2}\right\rangle}$ are of the order of $\sqrt{g}$ and occur at voltages of the order of $g V_{c}$. Thus, the regions of negative differential resistance predicted by LK in the high-voltage regime do not appear.

Section $\mathrm{V}$ is devoted to a comparison with experimental data of Refs. 10 and 11. Our results are summarized in Sec. VI. Technical details of the calculations are presented in several Appendixes.

\section{NONINTERACTING ELECTRONS}

We consider a quasi-one-dimensional (Q1D) metallic sample (a wire) of length $L$ attached to two reservoirs with a voltage difference $V$. In order to calculate the conductance variance in the strongly nonequilibrium regime, i.e., at a large bias voltage $V$, we employ the Keldysh technique (see Ref. 14; for a review see Ref. 15). In the Keldysh formalism the ensemble averaged current can be represented with the help of the ensemble averaged diagonal Keldysh function $\left\langle G_{\epsilon}^{K}(x)\right\rangle \equiv\left\langle G_{\epsilon}^{K}(x, x)\right\rangle=2 \pi i \nu\left[2 n_{\epsilon}(x)-1\right]$, where $n_{\epsilon}$ is the electron distribution function

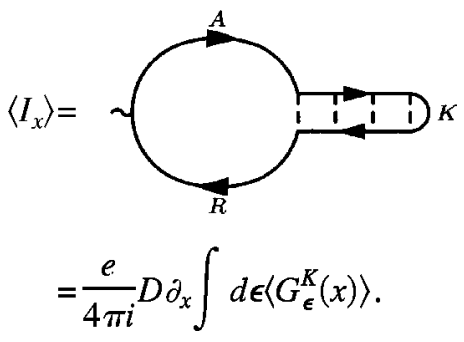

Here $D$ is the diffusion constant, $\nu$ is the density of states, $x$ is the coordinate along the wire, $R, A$, and $K$ denote the retarded, advanced, and Keldysh Green's functions respectively, and the wiggly line denotes the external current vertex, with which an operator $e \hat{v}_{x}$ is associated. The derivation of Eq. (1) is outlined in Appendix A. The diagrams for current fluctuations can be obtained by connecting two current diagrams by impurity ladders in all possible ways. We get the following six diagrams:
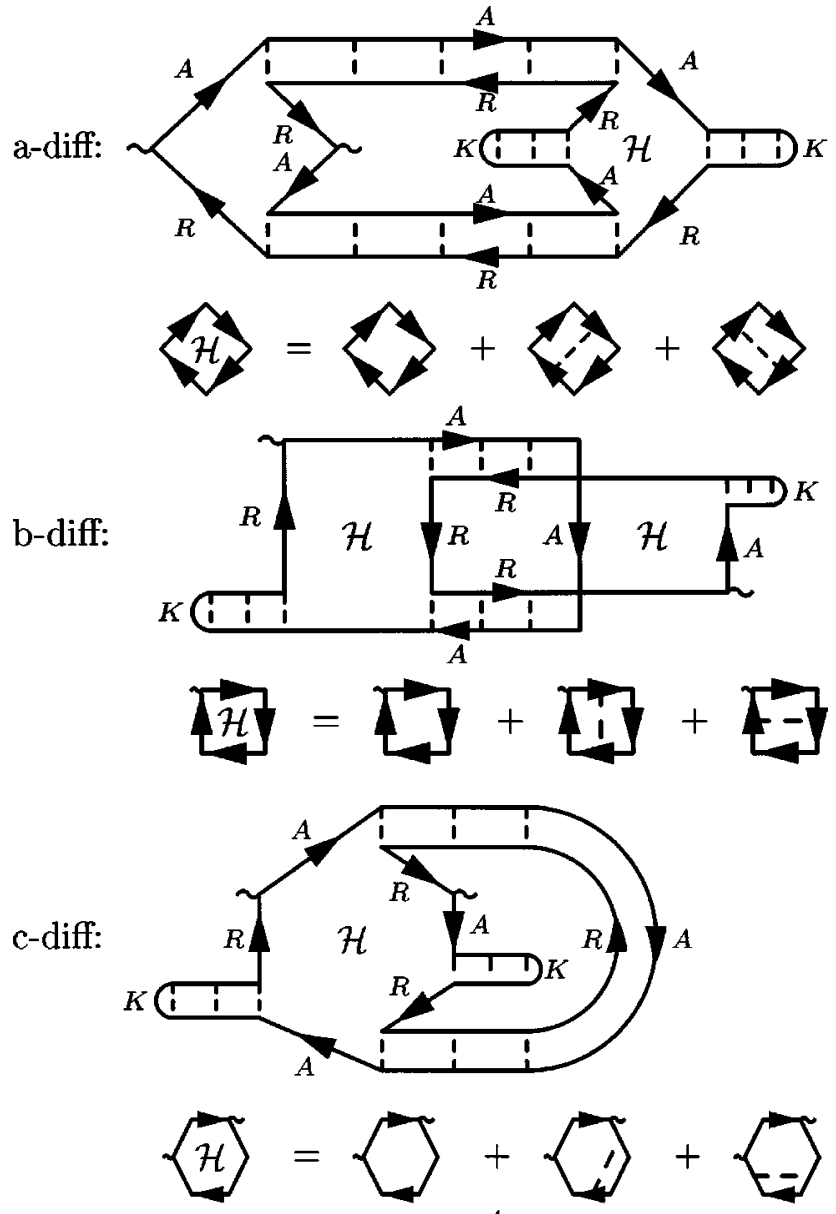

a-coop:
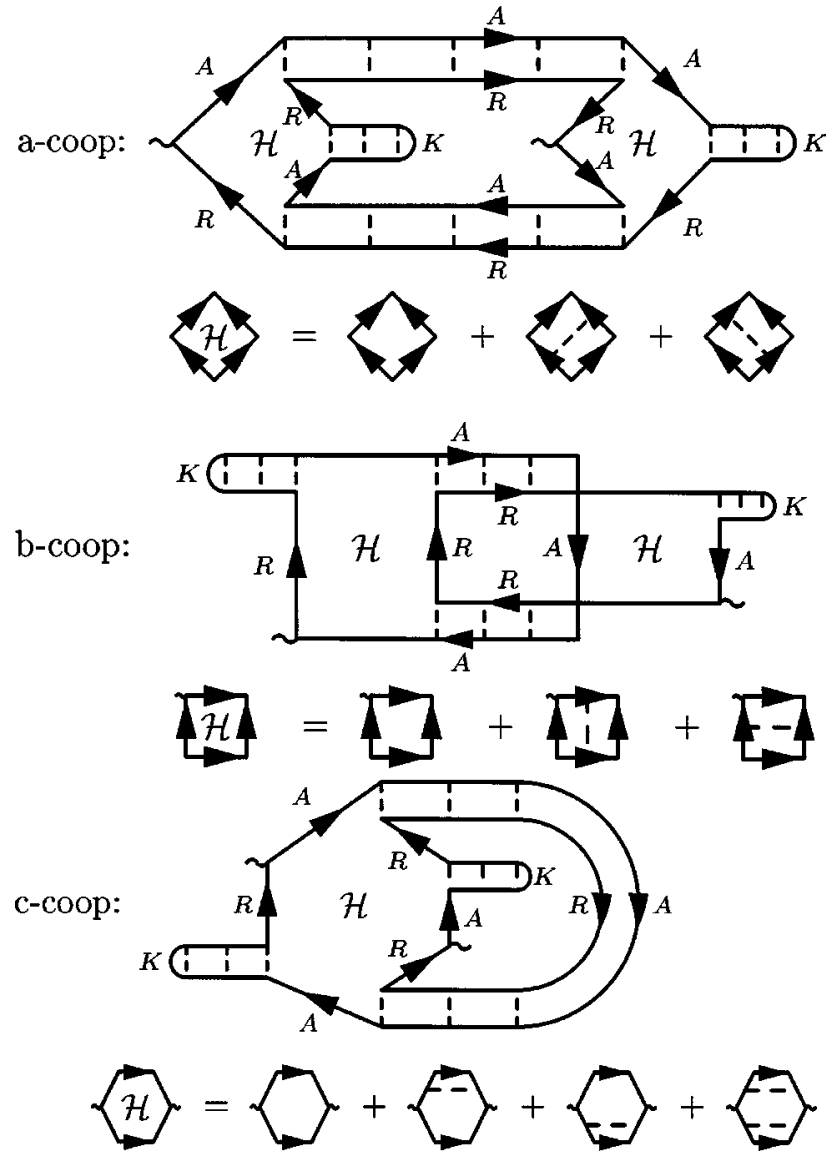
As usual in the impurity diagram technique, the diagrams consist of electronic vertices (Hikami boxes) connected by diffusons and Cooperons. The Hikami boxes take into account the possibility of inserting additional impurity lines connecting electronic Green functions of the same type (retarded with retarded, or advanced with advanced) without crossings. For the hexagonal vertices of the diagrams c-diff and c-coop, only the insertions contributing to the leading (zeroth) order in the external momenta are shown. Evaluating all the diagrams (the technical details of the calculation are presented in Appendix B), we get the following result for the correlation function of mesoscopic fluctuations of the current: ${ }^{16}$

$$
\begin{aligned}
\left\langle\delta I\left(V_{1}\right) \delta I\left(V_{2}\right)\right\rangle= & -\left(\frac{1}{2 \pi \nu}\right)^{2} \int d \epsilon_{1} d \epsilon_{2} \int \frac{d x_{1} d x_{2}}{L^{2}} \\
& \times \frac{\partial}{\partial x_{1}}\left\langle G_{\epsilon_{1}}^{K}\left(x_{1}\right)\right\rangle \frac{\partial}{\partial x_{2}}\left\langle G_{\epsilon_{2}}^{K}\left(x_{2}\right)\right\rangle \\
& \times\left[2\left|\Pi_{\epsilon_{1}-\epsilon_{2}}\left(x_{1}, x_{2}\right)\right|^{2}+\operatorname{Re} \Pi_{\epsilon_{1}-\epsilon_{2}}^{2}\left(x_{1}, x_{2}\right)\right] .
\end{aligned}
$$

Here $\Pi$ is a rescaled diffusion propagator satisfying the equation

$$
\left\{\partial_{x}^{2}+\frac{i \omega}{D}+\frac{i e}{D}\left[\phi_{1}(x)-\phi_{2}(x)\right]\right\} \Pi_{\omega}\left(x, x^{\prime}\right)=-\delta\left(x-x^{\prime}\right)
$$

with the boundary conditions that $\Pi=0$ if any of the coordinates $x, x^{\prime}$ is either 0 or $L$, and $\phi_{1,2}=(x / L) V_{1,2}$ are the potentials corresponding to the bias voltages $V_{1,2}$. Equation (2) is written for spinless electrons and unbroken time-reversal symmetry. Alternatively, it is valid for spinful electrons with a strong spin-orbit interaction, when only the spin-singlet channel contributes. If the spin-rotation symmetry is preserved, Eq. (2) should be multiplied by a factor 4 . If timereversal symmetry is broken, Eq. (2) should be multiplied by an extra factor of $1 / 2$. It is worth emphasizing that, when the complete set of diagrams is taken into account, they combine in such a way that only the spatial derivative of the Keldysh function enters the result, Eq. (2). This is because only the electrons in the energy window $V$ contribute to the transport. This property, which does not hold for individual diagrams but only for the sums of the diffuson or Cooperon diagrams, can serve as a useful check of the calculation.

We assume first that the temperature of reservoirs is sufficiently low, $T \ll e V_{c}$ [or, equivalently, the thermal diffusion length $L_{T}=(D / T)^{1 / 2}$ is much larger than the system size], so that we can set $T=0$. In other words, we will assume that at low voltage the system is in the regime of universal conductance fluctuations. The effect of a finite temperature, $T \gtrsim e V_{c}$, will be analyzed in Sec. III.

When a Q1D wire of length $L$ is connected to two perfect reservoirs with different Fermi energies, the electron distribution function in the wire will not be a Fermi function, but have a double-step shape. In the Keldysh formalism, this follows immediately from the kinetic equation for the average Keldysh function,

$$
D \nabla^{2}\left\langle G_{\epsilon}^{K}(x)\right\rangle=0
$$

with the boundary conditions

$$
\left\langle G_{\epsilon}^{K}(x)\right\rangle=-2 \pi i \nu \times\left\{\begin{array}{cc}
1-2 f(\epsilon), & x=0 \\
1-2 f(\epsilon-e V), & x=L,
\end{array}\right.
$$

where $f$ is the Fermi function. This equation implies that the Keldysh function is a linear function of the coordinate,

$$
\left\langle G_{\epsilon}^{K}(x)\right\rangle=-2 \pi i \nu\left\{1-2 f(\epsilon)+2 \frac{x}{L}[f(\epsilon)-f(\epsilon-e V)]\right\} .
$$

This yields (including a factor 4 to account for the spin)

$$
\left\langle\delta I\left(V_{1}\right) \delta I\left(V_{2}\right)\right\rangle=16 V_{c}^{2} \int_{0}^{V_{1} / V_{c}} d z_{1} \int_{0}^{V_{2} / V_{c}} d z_{2} \Xi_{z_{1}-z_{2}},
$$

where

$$
\begin{aligned}
\Xi_{z} & =\int_{0}^{1} d y_{1} d y_{2}\left[2\left|\Pi_{z}\left(y_{1}, y_{2}\right)\right|^{2}+\operatorname{Re} \Pi_{z}^{2}\left(y_{1}, y_{2}\right)\right] \\
& \equiv \operatorname{Tr}\left[2\left|\Pi_{z}\right|^{2}+\operatorname{Re} \Pi_{z}^{2}\right]
\end{aligned}
$$

and

$$
\left(\partial_{y}^{2}+i z+i \alpha y\right) \Pi_{z}\left(y, y^{\prime}\right)=-\delta\left(y-y^{\prime}\right) .
$$

Here we have introduced dimensionless variables $y=x / L$, $z=\omega / e V_{c}$, and $\alpha=\left(V_{1}-V_{2}\right) / V_{c}$.

To get the fluctuations of the differential conductance, we differentiate Eq. (7) with respect to both voltages and set $V_{1}=V_{2} \equiv V$. The result has the form (we set $T=0$ as discussed above)

$$
\langle\delta g \delta g\rangle=\langle\delta g \delta g\rangle_{0}+\langle\delta g \delta g\rangle_{1}+\langle\delta g \delta g\rangle_{2},
$$

where

$$
\begin{gathered}
\langle\delta g(V) \delta g(V)\rangle_{0}=\left.16 \Xi_{0}\right|_{\alpha=0}=\frac{8}{15}, \\
\langle\delta g(V) \delta g(V)\rangle_{1}=\left.32 \int_{0}^{V / V_{c}} d z \frac{\partial}{\partial \alpha} \Xi_{z-V / V_{c}}\right|_{\alpha=0}, \\
\langle\delta g(V) \delta g(V)\rangle_{2}=-\left.16 \int_{0}^{V / V_{c}} d z_{1} d z_{2} \frac{\partial^{2}}{\partial \alpha^{2}} \Xi_{z_{1}-z_{2}}\right|_{\alpha=0} .
\end{gathered}
$$

Let us now discuss the physical meaning of the three contributions (11)-(13) to the variance of the differential conductance. Consider first the low-voltage (linear) regime $V \ll V_{c}$. In this case, the only effect of a voltage increment $d V$ is to change the chemical potential in the reservoirs, and thus to make the states with energies in the window $d V$ available for electron transmission. The corresponding conductance fluctuations are given by the term (11), reproducing the wellknown UCF result, ${ }^{3}\left\langle\delta g^{2}\right\rangle=8 / 15$. The two other terms vanish at low voltages and thus describe truly nonequilibrium effects. Indeed, out of equilibrium the effect of the applied voltage is not only to change the chemical potential in the 
reservoirs, but also to alter the distribution of electric field in the sample. The shift $d V$ of the voltage induces the variation $d \phi(x)=(x / L) d V$ of the electrostatic potential, and this variation affects all the electrons in the energy window $V$ contributing to the current. This is the origin of the third term, Eq. (13). The second one, Eq. (12), is the cross-term due to correlations between the above two random contributions to $d I / d V$.

The following comment is in order here. When considering the conductance in terms of the scattering-theory formalism, one often replaces the problem by that of noninteracting electrons with different chemical potentials (or, equivalently, different concentrations) in the reservoirs. While the contribution $\langle\delta g \delta g\rangle_{0}$ is correctly reproduced in this way, the other two terms would be lost. What is not taken into account by such a noninteracting picture is that, for a system size much larger than the screening length, the main effect of changing the (electro-) chemical potential is not in altering the concentration but rather in changing the electrostatic potential in the reservoirs and thus modifying the potential profile in the wire.

To evaluate the derivatives in Eqs. (12) and (13), we expand $\Pi$ up to second order in $\alpha$,

$$
\Pi_{z}=\Pi_{z}^{(0)}+i \alpha \Pi_{z}^{(0)} y \Pi_{z}^{(0)}-\alpha^{2} \Pi_{z}^{(0)} y \Pi_{z}^{(0)} y \Pi_{z}^{(0)}+O\left(\alpha^{3}\right),
$$

where $\Pi_{z}^{(0)} \equiv\left(-\partial_{y}^{2}-i z\right)^{-1}$, and use the representation diagonalizing $\Pi_{z}^{(0)}$,

$$
\left(-\partial_{y}^{2}-i z\right)=\sum_{n>0} \lambda_{n}|n\rangle\langle n|,
$$

where $|n\rangle=\sqrt{2} \sin (n \pi y)$ and $\lambda_{n}=(n \pi)^{2}-i z$. The matrix elements of $y$ in this representation are given by

$$
\begin{gathered}
\langle n|y| m\rangle=\frac{4\left[(-1)^{m+n}-1\right] m n}{(m-n)^{2}(m+n)^{2} \pi^{2}}, \quad n \neq m \\
\langle n|y| n\rangle=\frac{1}{2} .
\end{gathered}
$$

We first find the asymptotic behavior of $\left\langle\delta g^{2}\right\rangle$ in the limit $V \gg V_{c}$. It is easy to see that the leading contribution is of the form $V / V_{c}$ and is governed by Eq. (13). Furthermore, only the term proportional to $|\Pi|^{2}$ should be retained in $\Xi_{z}$, Eq. (8), to find the asymptotics. Changing the variables $z_{1}, z_{2}$ to $Z=\left(z_{1}+z_{2}\right) / \sqrt{2}, z=\left(z_{1}-z_{2}\right) / \sqrt{2}$, using the expansion (14), and evaluating the integrals via the residue theorem, we get

$$
\left\langle\delta g^{2}\right\rangle_{V \gg V_{c}}=-\left.32 \frac{V}{V_{c}} \int d z \frac{\partial^{2}}{\partial \alpha^{2}} \operatorname{Tr}\left|\Pi_{z}\right|^{2}\right|_{\alpha=0}=c_{1} \frac{V}{V_{c}},
$$

where

$$
\begin{aligned}
c_{1} & =64 \sum_{m, n>0} \frac{1}{\pi^{5}}\left[\frac{1}{n^{4}\left(m^{2}+n^{2}\right)}-\frac{1}{m^{2} n^{2}\left(m^{2}+n^{2}\right)}\right]\langle n|y| m\rangle^{2} \\
& =7.785 \times 10^{-4} .
\end{aligned}
$$

Therefore, although the large- $V$ asymptotics is indeed of the form $V / V_{c}$ obtained by LK, the corresponding numerical co-

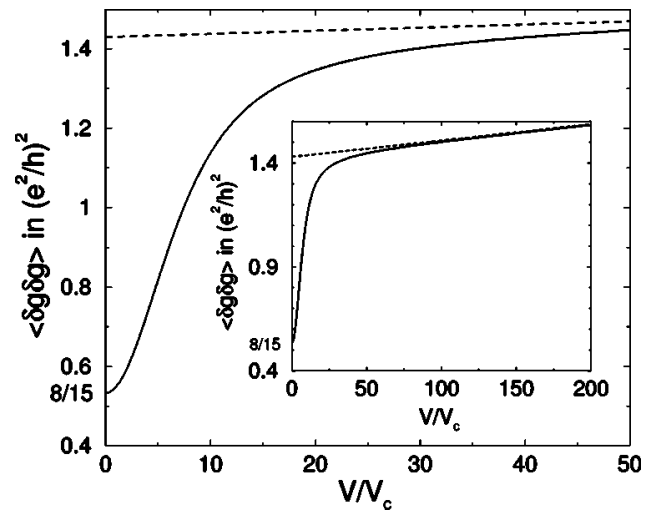

FIG. 1. Fluctuations $\left\langle\delta g^{2}\right\rangle$ of the differential conductance as a function of the bias voltage normalized to the Thouless energy, $V / V_{c}$. The inset shows the emergence of the linear behavior at very large $V / V_{c}$. The dashed line represents the asymptotic behavior, $\left\langle\delta g^{2}\right\rangle=8 / 15+c_{0}+c_{1} V / V_{c}$ with $c_{0}=0.8964$ and $c_{1}=7.785 \times 10^{-4}$.

efficient $c_{1}$ (not evaluated in Ref. 4) is very small.

We now turn to the evaluation of the full crossover behavior of $\left\langle\delta g^{2}\right\rangle$ as a function of $V / V_{c}$. It is more cumbersome, but can be done in a similar way; see Appendix C for details. The result is shown in Fig. 1. The striking feature of this plot is that the large- $V$ asymptotics (17) becomes applicable only at very large values of $V / V_{c}$. The emergence of the linear contribution with a coefficient given by Eq. (17) can be seen for $V / V_{c} \gtrsim 50$ but it becomes dominant only at $V / V_{c} \gtrsim 1000$. For $V / V_{c} \gtrsim 50$ the conductance fluctuations are well described by the asymptotic formula that includes, in addition to the linear term (17), a $V$-independent contribution (which is formally of the next order but numerically dominates up to $V / V_{c} \sim 1000$ ),

$$
\left\langle\delta g^{2}\right\rangle_{V \gg V_{c}}=\frac{8}{15}+c_{0}+c_{1} \frac{V}{V_{c}},
$$

with $c_{0}=0.8964$. On the other hand, this very slow increase of $\left\langle\delta g^{2}\right\rangle$ (barely distinguishable from a saturation) at large voltages is preceded by a broad crossover regime at $V / V_{c}$ $\lesssim 50$, where the conductance increases by roughly a factor of 3. In the experiments, the increase of $\left\langle\delta g^{2}\right\rangle$ is observed in the range $V / V_{c} \lesssim 200$. As is obvious from Fig. 1, this enhancement should be mainly attributed to the crossover regime rather than to the LK linear asymptotics. We will return to the comparison with experiment later, after having considered the effects of finite temperature and of the interactioninduced dephasing.

\section{FINITE TEMPERATURE}

In this section, we address the effect of finite temperature on the nonequilibrium conductance fluctuations. We assume that the temperature is not too high, so that at low voltages the inelastic scattering can be neglected [the dephasing length $L_{\phi}(T)$ is large compared to the system size]. The temperature, however, influences the result via the change of the electron distribution function in the reservoirs. At a finite temperature, Eqs. (11)-(13) are modified in the following 
way (here we switch back to dimensionful voltages for clarity):

$$
\begin{gathered}
\langle\delta g \delta g\rangle_{0}=\frac{16}{e^{2}} \int d \epsilon_{1} d \epsilon_{2} \Delta f_{1}^{\prime} \Delta f_{2}^{\prime} \Xi_{\epsilon_{1}-\epsilon_{2}}, \\
\langle\delta g \delta g\rangle_{1}=\frac{16}{e^{2}} \int d \epsilon_{1} d \epsilon_{2} \Delta f_{1}^{\prime} \Delta f_{2} \frac{\partial}{\partial V_{2}} \Xi_{\epsilon_{1}-\epsilon_{2}} \\
+\frac{16}{e^{2}} \int d \epsilon_{1} d \epsilon_{2} \Delta f_{1} \Delta f_{2}^{\prime} \frac{\partial}{\partial V_{1}} \Xi_{\epsilon_{1}-\epsilon_{2}}, \\
\langle\delta g \delta g\rangle_{2}=\frac{16}{e^{2}} \int d \epsilon_{1} d \epsilon_{2} \Delta f_{1} \Delta f_{2} \frac{\partial^{2}}{\partial V_{1} \partial V_{2}} \Xi_{\epsilon_{1}-\epsilon_{2}},
\end{gathered}
$$

where $\Delta f_{i}=f\left(\epsilon_{i}\right)-f\left(\epsilon_{i}+e V_{i}\right)$ ( $f$ is the Fermi function) and $\Delta f_{i}^{\prime}=\left(\partial / \partial V_{i}\right) \Delta f_{i}$. Again the derivatives are taken at $V_{1}=V_{2}$ $=V$.

Evaluation of the full crossover (i.e., the conductance fluctuations at arbitrary $V / V_{c}$ and $T / e V_{c}$ ) is too cumbersome. We thus restrict ourselves to the limit $T \gg e V_{c}$, when the temperature strongly affects the conductance fluctuations at low voltages (in the opposite limit, $T \ll e V_{c}$, the temperature is irrelevant for all voltages, so that the results of Sec. II apply). This condition is reasonably fulfilled in most of the relevant experiments, where the temperature is usually several times higher than the Thouless energy.

We consider now the two limits of low $\left(V \ll V_{c}\right)$ and high $\left(V \gg V_{c}\right)$ bias voltages. In the first case, $V / V_{c} \rightarrow 0$, only the voltage-independent contribution $\langle\delta g \delta g\rangle_{0}$ survives. For $T \gg e V_{c}$ the thermal smearing strongly suppresses this contribution compared to its zero-temperature (UCF) value,

$$
\langle\delta g \delta g\rangle_{0}=\frac{T \gg V_{c}}{9} \frac{8 \pi}{9} \frac{e V_{c}}{T} .
$$

In the opposite limit, when $e V, T \gg e V_{c}$ (the ratio $e V / T$ can be arbitrary), we find that the high-voltage behavior (19) is modified by the temperature in the following way:

$$
\langle\delta g \delta g\rangle=a\left(\frac{e V}{T}\right) c_{0}+b\left(\frac{e V}{T}\right) c_{1} \frac{V}{V_{c}}, V \gg V_{c}
$$

where the functions $a(e V / T)$ and $b(e V / T)$ have the form

$$
\begin{aligned}
& a\left(\frac{e V}{T}\right)=2 \int d \epsilon[f(\epsilon)-f(\epsilon+e V)]\left(-\frac{\partial f}{\partial \epsilon}\right) \\
&=\frac{\sinh (e V / T)-e V / T}{\cosh (e V / T)-1}, \\
& b\left(\frac{e V}{T}\right)=\frac{1}{e V} \int d \epsilon[f(\epsilon)-f(\epsilon+e V)]^{2}=\operatorname{coth} \frac{e V}{2 T}-\frac{2 T}{e V} .
\end{aligned}
$$

It is worth emphasizing that for any $T$ at sufficiently high bias voltages the $T=0$ result is recovered up to the missing offset of $8 / 15$.

Adding the term $\langle\delta g \delta g\rangle_{0}$, Eq. (23) to Eq. (24), we get an interpolation formula that is parametrically justified in the

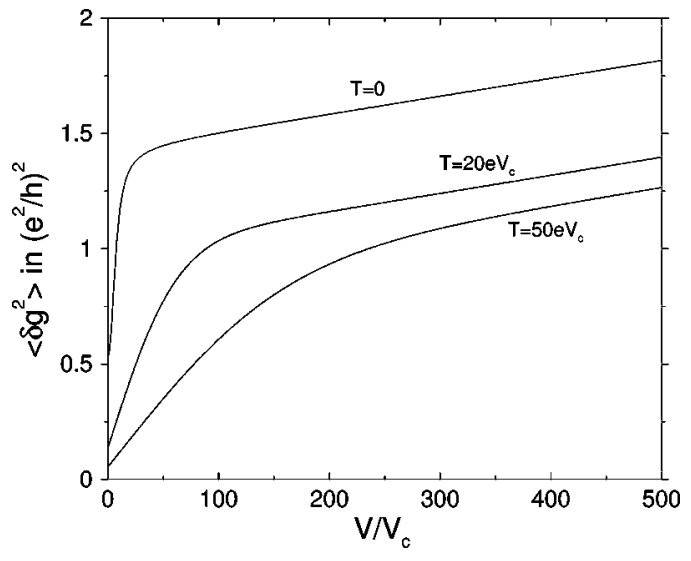

FIG. 2. The voltage dependence of the conductance fluctuations as given by the sum of Eqs. (23) and (24), for the temperatures $T=20 e V_{c}$ and $T=50 e V_{c}$. The zero- $T$ result of Fig. 1 is also shown for comparison. It is clearly seen that the coefficient of the asymptotic linear behavior is not changed by the temperature.

regime $V \gg V_{c}$ and has the correct limit at $V / V_{c} \rightarrow 0$. This formula can thus be used as a convenient approximation for $\langle\delta g \delta g\rangle$ in the full range of voltages at $T \gg e V_{c}$. The resulting voltage dependence of the conductance fluctuations is shown in Fig. 2 for the temperatures $T=20 e V_{c}$ and $T=50 e V_{c}$. We compare these theoretical results with experimental data in Sec. V below.

\section{DEPHASING BY ELECTRON-ELECTRON SCATTERING AT HIGH BIAS VOLTAGES}

Up to now, we have neglected the dephasing induced by the inelastic scattering processes, which is justified for not too high bias voltages $V$. With increasing $V$, the electronelectron scattering becomes stronger, and eventually leads to suppression of the conductance fluctuations. The corresponding theory is presented in this section. As in Sec. III, we will assume that the temperature at the reservoirs is sufficiently low (more precisely, $T \ll g e V_{c}$ ), so that the corresponding dephasing length is much larger than the system size, $L_{\phi}(T) \gg L$. In this situation, the dephasing will be entirely due to a high applied voltage.

In quasi-one-dimensional diffusive systems, dephasing is dominated by scattering processes with a small energy transfer ${ }^{12,13} \omega \ll T$, which allows one to replace the dynamically screened Coulomb interaction by a fluctuating classical potential. The same arguments apply in the presence of a high bias voltage, when the fluctuations of the electric field are characterized by the effective temperature $T_{\text {eff }}$ defined below. In view of the semiclassical character of the problem, we can use the Boltzmann-Langevin approach (see Refs. 17 and 18) to determine the correlation function of the fluctuating field. The fluctuation of the local current density $j$ can be expressed in the following way:

$$
\delta \boldsymbol{j}=-i \boldsymbol{q} D \delta \rho+\sigma \delta \boldsymbol{E}+\delta \boldsymbol{j}^{\mathrm{ext}},
$$

where the first term is the diffusion flow produced by the density fluctuation $\delta \rho$, the second one is due to the fluctua- 
tion $\delta \boldsymbol{E}$ of electric field ( $\sigma=e^{2} \nu D$ is the conductivity), and the last term represents extraneous (Langevin) current fluctuations induced by elastic scattering. At equilibrium, these correlations are found from the fluctuation-dissipation theorem. ${ }^{13}$ Out of equilibrium, they have been studied in the shot noise context. ${ }^{19}$ We only need the fluctuations of $x$ components of the current (those along the wire),

$$
\left\langle\delta j_{x}^{\mathrm{ext}}(x, t) \delta j_{x}^{\mathrm{ext}}\left(x^{\prime}, t^{\prime}\right)\right\rangle=2 \sigma T_{\mathrm{eff}} \delta\left(x-x^{\prime}\right) \delta\left(t-t^{\prime}\right),
$$

with the effective temperature $T_{\text {eff }}(x)$ given by

$$
T_{\text {eff }}=\int d \epsilon n_{\epsilon}(x)\left[1-n_{\epsilon}(x)\right],
$$

where $n_{\epsilon}(x)$ is the (in general, nonequilibrium) distribution function. This function itself is strongly voltage dependent.

For not too high $V$, the distribution function has a doublestep shape (6); for higher voltages the inelastic scattering processes lead to thermalization of electrons, and the distribution function assumes the Fermi shape with a local temperature. ${ }^{19}$ The boundary between the two regimes is determined by the energy relaxation time $\tau_{\epsilon}$ : no thermalization takes place for $e V_{c} \gg \tau_{\epsilon}^{-1}$. It is important that the dephasing is dominated by small energy transfers $\epsilon \ll T_{\text {eff }}$, which implies ${ }^{13}$ that the dephasing time $\tau_{\phi}$ is much shorter than the energy relaxation time $\tau_{\epsilon}$. Thus, even when the system enters, with increasing voltage, the regime of strong dephasing, $e V_{c}$ $\ll \tau_{\phi}^{-1}$, the energy relaxation still remains weak, $e V_{c} \gg \tau_{\epsilon}^{-1}$, in a parametrically broad range of voltages. From now on, we consider this regime, and find for the effective temperature

$$
T_{\text {eff }}(x)=e V \frac{x}{L}\left(1-\frac{x}{L}\right) .
$$

This effective temperature is position dependent, taking its maximum in the middle of the wire and approaching zero at the contacts with the reservoirs. Using the continuity equation,

$$
\delta \rho=\frac{q}{\omega} \delta j,
$$

and the relation between fluctuations of the electric field $\boldsymbol{E}=-\boldsymbol{\nabla} \phi$ and the density,

$$
e \delta \phi(\boldsymbol{q})=\frac{\delta \rho(\boldsymbol{q})}{e} U^{(0)}(q),
$$

where $U^{(0)}\left(\left|\boldsymbol{r}-\boldsymbol{r}^{\prime}\right|\right)=e^{2} /\left|\boldsymbol{r}-\boldsymbol{r}^{\prime}\right|$ is the bare Coulomb interaction, one finds

$$
\delta j_{x}=\frac{-i \omega}{-i \omega+D q^{2}\left[1+\nu U^{(0)}(q)\right]} \delta j_{x}^{\text {ext }} .
$$

Since for Coulomb interaction in metals $\nu U^{(0)}(q) \gg 1$, Eq. (27) reduces to

$$
\sigma \delta E_{x}(q)=-\delta j_{x}^{\text {ext }}(q), \quad q \neq 0 .
$$

The $q=0$ component of $\delta E_{x}=-\partial_{x} \phi$ is zero due to the boundary conditions: there are no potential fluctuations at the perfect reservoirs. Therefore, the electric field fluctuations in the coordinate space are given by

$$
\delta E_{x}(x)=-\frac{1}{\sigma}\left[\delta j_{x}^{\mathrm{ext}}(x)-\frac{1}{L} \int d x \delta j_{x}^{\mathrm{ext}}(x)\right] .
$$

Using Eq. (30), we obtain the correlator of the electric fields,

$$
\begin{aligned}
\left\langle\delta E_{x}(x) \delta E_{x}\left(x^{\prime}\right)\right\rangle= & \frac{2}{\sigma}\left[T_{\mathrm{eff}}(x) \delta\left(x-x^{\prime}\right)-\frac{1}{L} T_{\mathrm{eff}}(x)-\frac{1}{L} T_{\mathrm{eff}}\left(x^{\prime}\right)\right. \\
& \left.+\frac{1}{L^{2}} \int d \widetilde{x} T_{\mathrm{eff}}(\widetilde{x})\right]
\end{aligned}
$$

The correlator of the potential can be found by spatial integration. We introduce dimensionless variables $y=x / L$, $\tau=e V_{c} t, \theta=\phi / e V_{c}$, and find

$$
\begin{aligned}
\left\langle\theta(y, \tau) \theta\left(y^{\prime}, \tau^{\prime}\right)\right\rangle= & \frac{2 V}{3 V_{c} g} \delta\left(\tau-\tau^{\prime}\right) y_{<}\left(1-y_{>}\right) \\
& \times\left[y(1-y)+y^{\prime}\left(1-y^{\prime}\right)-\frac{\left(y_{>}-y_{<}\right)}{2}\right],
\end{aligned}
$$

where $y_{<}=\min \left(y, y^{\prime}\right)$ and $y_{>}=\max \left(y, y^{\prime}\right)$.

As can be seen from Eq. (37), the dimensionless parameter $V / V_{c} g$ is a measure for the importance of inelastic processes. Therefore, in the presence of the Coulomb interaction, the results of Sec. II hold under the condition $V \leqq V_{c} g$. This condition excludes, in particular, voltages of order $V \sim V_{c} g^{2}$ that are required to reach the regime where the fluctuating differential resistance would become negative in the absence of dephasing.

As we noted in the previous section, the part $\sim|\Pi|^{2}$ of Eq. (13) dominates at high voltages, so that we can neglect Re $\Pi^{2}$. Following the approach of Refs. 13 and 20, we express $\Pi$ as a path integral,

$$
\begin{aligned}
\operatorname{Tr}\left|\Pi_{z}\right|^{2}= & \int_{0}^{1} d y_{1} d y_{2} \int_{0}^{\infty} d \tau_{1} d \tau_{2} \mathrm{e}^{i z\left(\tau_{1}-\tau_{2}\right)} \int_{\xi_{1}(0)=y_{2}}^{\xi_{1}\left(\tau_{1}\right)=y_{1}} \mathcal{D} \xi_{1}\left(t_{1}\right) \\
& \times \int_{\xi_{2}(0)=y_{2}}^{\xi_{2}\left(\tau_{2}\right)=y_{1}} \mathcal{D} \xi_{2}\left(t_{2}\right) \exp \left\{\int_{0}^{\tau_{1}} d t_{1}\right. \\
& \times\left[-\frac{\dot{\xi}_{1}^{2}}{4}-\frac{i\left(V_{1}-V_{2}\right)}{V_{c}} \xi_{1}\left(t_{1}\right)-i \theta\left(\xi_{1}\left(t_{1}\right), t_{1}\right)\right] \\
& +\int_{0}^{\tau_{2}} d t_{2}\left[-\frac{\dot{\xi}_{2}^{2}}{4}+\frac{i\left(V_{1}-V_{2}\right)}{V_{c}} \xi_{2}\left(t_{2}\right)\right. \\
& \left.\left.+i \theta\left(\xi_{2}\left(t_{2}\right), t_{2}\right)\right]\right\} .
\end{aligned}
$$

We now perform the averaging over the random fields by using Eq. (37). When dephasing is strong, conductance fluctuations are dominated by pairs of paths which stay close to each other, $\left|\xi_{1}(t)-\xi_{2}(t)\right| \ll 1$. We thus expand the terms in the action representing the dephasing in $\left|\xi_{1}(t)-\xi_{2}(t)\right|$, which yields 


$$
\begin{aligned}
\left\langle\operatorname{Tr}\left|\Pi_{z}\right|^{2}\right\rangle= & \int_{0}^{1} d y_{1} d y_{2} \int_{0}^{\infty} d \tau_{1} d \tau_{2} \mathrm{e}^{i z\left(\tau_{1}-\tau_{2}\right)} \int_{\xi_{1}(0)=y_{2}}^{\xi_{1}\left(\tau_{1}\right)=y_{1}} \mathcal{D} \xi_{1}\left(t_{1}\right) \int_{\xi_{2}(0)=y_{2}}^{\xi_{2}\left(\tau_{2}\right)=y_{1}} \mathcal{D} \xi_{2}\left(t_{2}\right) \exp \left\{\int_{0}^{\tau_{1}} d t_{1}\left[-\frac{\dot{\xi}_{1}^{2}}{4}-\frac{i\left(V_{1}-V_{2}\right)}{V_{c}} \xi_{1}\left(t_{1}\right)\right]\right. \\
& \left.+\int_{0}^{\tau_{2}} d t_{2}\left[-\frac{\dot{\xi}_{2}^{2}}{4}+\frac{i\left(V_{1}-V_{2}\right)}{V_{c}} \xi_{2}\left(t_{2}\right)\right]-\frac{2 V}{V_{c} g} F\left(y_{2}\right)\left|\tau_{1}-\tau_{2}\right|-\frac{2 V}{V_{c} g} \int_{0}^{\min \left(\tau_{1}, \tau_{2}\right)} d t\left|\xi_{1}(t)-\xi_{2}(t)\right| y_{2}\left(1-y_{2}\right)\right\},
\end{aligned}
$$

$$
F(y)=\frac{2}{3} y^{2}(1-y)^{2} .
$$

Note that the factors $F\left(y_{2}\right)$ and $y_{2}\left(1-y_{2}\right)$ in the dephasing terms could be equally well written as $F\left(y_{1}\right)$ and $y_{1}\left(1-y_{1}\right)$, respectively, since we assumed that the distance between $y_{1}$ and $y_{2}$ is much smaller than their distance to the reservoirs, $\left|y_{1}-y_{2}\right| \ll y_{1},\left(1-y_{1}\right)$. We will have to check the consistency of this assumption afterwards.

The last two terms in the exponent of Eq. (39) represent the dephasing effects. The first of them is the dephasing during a small time difference $\left|\tau_{1}-\tau_{2}\right|$ between the time extensions of both diffuson trajectories, while the second one is the dephasing during the motion of both diffusons. Since after the $z$ integration the characteristic values of $\left|\tau_{1}-\tau_{2}\right|$ are of order $V_{c} / V$, the first term gives a contribution of order $1 / g$ to the exponent and thus can be neglected.

Employing the Fourier transform,

$$
\Xi_{z}=\int d \tau \mathrm{e}^{\mathrm{i} z \tau} \tilde{\Xi}(\tau), \int d z \Xi_{z}=2 \pi \widetilde{\Xi}(0) .
$$

We can write the important part of Eq. (13) (the part containing $|\Pi|^{2}$ ) for $V \gg g V_{c}$ as

$$
\langle\delta g \delta g\rangle_{2}=\left.32 \pi V V_{c} \frac{\partial^{2}}{\partial V_{1} \partial V_{2}} \widetilde{\Xi}(0)\right|_{V_{1}=V_{2}=V},
$$

where $\widetilde{\Xi}(0)$ is twice Eq. (39) with $\tau_{1}$ set to $\tau_{2}$.

By changing to new variables $\zeta_{1}=\left(\xi_{1}+\xi_{2}\right) / \sqrt{2}, \quad \zeta_{2}=\left(\xi_{1}\right.$ $\left.-\xi_{2}\right) / \sqrt{2}$ we can perform the path integral over $\zeta_{1}$, which is not affected by the random fields and just yields $\Theta(\tau) / \sqrt{2}$. Then

$$
\langle\delta g \delta g\rangle_{2}=\left.32 \sqrt{2} \pi V V_{c} \frac{\partial^{2}}{\partial V_{1} \partial V_{2}} \int_{0}^{1} d y \int_{0}^{\infty} d \tau I_{y}(0, \tau)\right|_{V_{1,2}=V},
$$

where $I_{y}(\zeta, \tau)$ obeys the equation

$$
\begin{aligned}
& {\left[\partial_{\tau}-\partial_{\zeta}^{2}+i \sqrt{2} \frac{\left(V_{1}-V_{2}\right)}{V_{c}} \zeta+\frac{2 V}{V_{c} g} \sqrt{2} y(1-y)|\zeta|\right] I_{y}(\zeta, \tau)} \\
& \quad=\delta(\zeta) \delta(\tau) .
\end{aligned}
$$

This can be made dimensionless by choosing new variables $t=p^{2 / 3} \tau, \eta=p^{1 / 3} x, \mathcal{I}=p^{-1 / 3} I$, and $v=i \sqrt{2}\left(V_{1}-V_{2}\right) / V_{c} p$, where $p=\sqrt{2} y(1-y) 2 V / V_{c} g$. Performing the time integration over $t$, we get

$$
\langle\delta g \delta g\rangle_{2}=\left.8 \pi\left(\frac{V_{c}}{V}\right)^{4 / 3} g^{7 / 3} \frac{\partial^{2}}{\partial v^{2}} Q(0)\right|_{V=0} \int_{0}^{1} \frac{d y}{[y(1-y)]^{7 / 3}},
$$

where $Q$ satisfies $\left(-\partial_{\eta}^{2}+|\eta|+v \eta\right) Q(\eta)=\delta(\eta)$. We see that the resulting integral diverges at the upper and lower limits corresponding to the vicinity of the reservoirs. The reason for this divergence is as follows: Near the reservoirs the expansion of the action in $\left|\xi_{1}(t)-\xi_{2}(t)\right|$, used to derive Eq. (39), ceases to be valid, since the characteristic values of $\mid \xi_{1}(t)$ $-\xi_{2}(t) \mid$ determined by the local value of the dephasing length $L_{\phi}(y)$ become of order of $y$ or $(1-y)$. The above divergence shows that these small vicinities of reservoirs (where the effective temperature approaches zero and the dephasing length is larger than that in the rest of the wire) in fact dominate the conductance fluctuations.

The exact calculation thus requires using the full form of the correlation function (37), which makes the problem much more cumbersome, leading to a two-dimensional Schrödinger-type differential equation rather than to the onedimensional equation (44). We can get, however, the result, up to a numerical prefactor of order unity, in a simpler way. Indeed, as follows from the consideration above, the integral must be cut off at a distance $L y_{c}$ to the reservoirs, which is of the order of the local phase-breaking length, yielding the self-consistency condition

$$
L y_{c} \sim L_{\phi}\left(y_{c}\right)=\sqrt{D \tau_{\phi}\left[T_{\text {eff }}\left(y_{c}\right)\right]},
$$

where $T_{\text {eff }}(y)=e V y(1-y)$ and $\tau_{\phi}\left[T_{\text {eff }}\right] \sim\left(D \nu^{2} / T_{\text {eff }}^{2}\right)^{1 / 3}$. This yields a cutoff

$$
y_{c} \sim\left(\frac{V_{c} g}{V}\right)^{1 / 4} .
$$

Therefore, the amplitude of conductance fluctuations, which are dominated by the coherent segments within the distance $L y_{c}$ to the contacts, is given by

$$
\langle\delta g \delta g\rangle_{2} \sim g^{2} \frac{V_{c}}{V}, \frac{V}{V_{c}} \gg g .
$$

Thus, the variance of the conductance shows a maximum at $V / V_{c} \sim g$ and then decays according to the $1 / V$ power law.

Strictly speaking, in order to show rigorously that the asymptotic suppression at high bias voltages is given by this power law, we still have to check that $\langle\delta g \delta g\rangle_{1}$ and $\langle\delta g \delta g\rangle_{0}$ remain smaller than $\langle\delta g \delta g\rangle_{2}$ with increasing voltage. This turns out to be the case, as is shown in Appendix D. 
A natural question to be asked at this point is whether the result (48) can be obtained from simple qualitative arguments. Indeed, we know that for the case of the linearresponse transport, when the dephasing length $L_{\phi}$ is governed by the temperature $L_{\phi}=L_{\phi}(T)$, the conductance fluctuations of a wire in the high-temperature regime, $L_{\phi}(T) \ll L$, can be estimated in the following way. The system is split into segments of the length $L_{\phi}$. The electrons within each of the segments remain phase coherent, yielding fluctuations of the conductance of the segment, $\left\langle\delta g^{2}\left(L_{\phi}\right)\right\rangle$ $\sim D / L_{\phi}^{2} T$ [see Eq. (23)]. A fluctuation $\delta g\left(L_{\phi}\right)$ of the conductance of a segment induces a fluctuation in the total conductance

$$
\delta g \sim\left(L_{\phi} / L\right)^{2} \delta g\left(L_{\phi}\right) .
$$

Adding incoherently fluctuations of all $L / L_{\phi}$ segments, one gets

$$
\left\langle\delta g^{2}\right\rangle \sim\left(L_{\phi} / L\right)^{3}\left\langle\delta g^{2}\left(L_{\phi}\right)\right\rangle \sim \frac{D}{L^{2} T} \frac{L_{\phi}}{L} .
$$

This agrees with the diagrammatic calculation ${ }^{3}$ (using $L_{\phi}$ as a phenomenological parameter), as well as with the path integral calculation, ${ }^{20}$ yielding the dephasing length $L_{\phi}(T)$ induced by the electron-electron scattering, $L_{\phi}(T)$ $\sim\left(D^{2} \nu / T\right)^{1 / 3}$.

A generalization of this consideration on the strongly nonequilibrium setup was attempted in Ref. 10 but the obtained result (mentioned in Sec. I) was different from Eq. (48). The arguments presented in Ref. 10 miss the following two nontrivial properties of the nonequilibrium dephasing, which distinguish it from its equilibrium, finite- $T$ counterpart. First, as we have shown above, the dephasing length in nonequilibrium is strongly position-dependent, and the conductance fluctuations are dominated by the segments of the wire adjacent to the reservoirs. Therefore, representing the wire as an incoherent series of $L / L_{\phi}(\mathrm{eV})$ equal segments of a length $L_{\phi}(e V)$ is not justified. Second, the conductance fluctuations in a segment of a length $L_{\phi}$ cannot be obtained simply using the results for a coherent sample of a length $L_{\phi}$ subject to a voltage $V\left(L_{\phi}\right)=V L_{\phi} / L$. This is because the nonequilibrium distribution function in the segment $L_{\phi}$ is not determined by a single parameter $V\left(L_{\phi}\right)$ but rather is governed by two parameters, $V$ and $L_{\phi} / L$.

We are now going to show that when these peculiarities are properly taken into account, the qualitative consideration does reproduce the result (48). Consider a coherent segment of a length $L_{\phi}$ (to be specified later). Generalizing the consideration in Sec. III, the variance of its differential conductance can be estimated as

$$
\left\langle\delta g^{2}\left(L_{\phi}\right)\right\rangle \sim \frac{1}{e V_{c}\left(L_{\phi}\right)} \int d \epsilon\left[\Delta f_{L_{\phi}}(\epsilon)\right]^{2},
$$

where $e V_{c}\left(L_{\phi}\right)=D / L_{\phi}^{2}$ is the Thouless energy corresponding to the segment $L_{\phi}$, and $\Delta f_{L_{\phi}}(\epsilon)$ is the difference between the distribution function at the left and right boundaries of this segment. It is easy to see that $\Delta f_{L_{\phi}}(\epsilon)$ is equal to $L_{\phi} / L$ in the

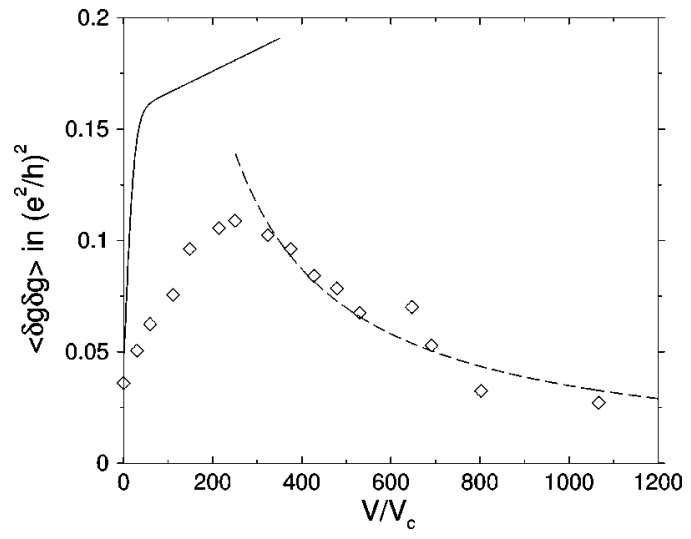

FIG. 3. Comparison of our results with experimental data taken from Refs. 10 and 11. The diamonds are the data from Fig. 5.4 of Ref. 11. The solid line represents the interpolation formula obtained by adding Eqs. (23) and (24), multiplied by $1 / 8$ to account for spin-orbit interaction and broken time-reversal symmetry. The dashed line represents the asymptotic suppression, Eq. (48), with the numerical coefficient chosen from the fit to the data.

energy window of the width $e V$, and is zero outside this window. This yields

$$
\left\langle\delta g^{2}\left(L_{\phi}\right)\right\rangle \sim \frac{1}{e V_{c}\left(L_{\phi}\right)} e V\left(\frac{L_{\phi}}{L}\right)^{2} \sim \frac{V}{V_{c}}\left(\frac{L_{\phi}}{L}\right)^{4} .
$$

According to Eq. (49), this induces fluctuations of the wire conductance of the magnitude

$$
\left\langle\delta g^{2}\right\rangle \sim\left(\frac{L_{\phi}}{L}\right)^{8} \frac{V}{V_{c}} .
$$

Finally, using that $L_{\phi}=L y_{c}$ with $y_{c}$ given by Eq. (47), we get $\left\langle\delta g^{2}\right\rangle \sim g^{2} V_{c} / V$, thus reproducing the result (48) of the pathintegral calculation.

\section{COMPARISON WITH EXPERIMENT}

In this section we compare our results with the measurements presented in Refs. 10 and 11. There, magnetoconductance traces of a gold wire with a length $L=1.5 \mu \mathrm{m}$ and a Thouless energy of $e V_{c} \equiv D / L^{2}=3.4 \mu \mathrm{eV}$ were taken at $T=300 \mathrm{mK}$ over a voltage range up to $V \simeq 3.7 \mathrm{mV}$. This yields $T / e V_{c} \simeq 7.6$ and the maximum value of $V / V_{c} \approx 1000$. The dimensionless conductance of the sample was $g \approx 1400$.

Figure 3 shows our results for both the noninteracting limit (not too high voltages) and the strong-dephasing limit (very high voltages), compared with the measured data. It is seen that there is a reasonably good agreement between the theory and the experiment in the overall shape of $\langle\delta g \delta g\rangle\left(V / V_{c}\right)$ and in the magnitude of its enhancement at the maximum.

The main deviation is that the initial increase of the conductance variance is considerably less steep than on the theoretical curve. This discrepancy can be partly attributed to the following factors. First, we used for the full curve in Fig. 3 the formula (24), which takes into account the crossover at $e V \sim T$ but not the crossover at $V \sim V_{c}$. Though the tempera- 
ture was larger than $e V_{c}$ by a factor $\sim 8$ in the experiment, we know from Sec. II that the $V / V_{c}$ crossover region is rather broad, extending up to $V / V_{c} \sim 50$. Thus the $V / V_{c}$ crossover is expected to overlap with the $e V / T$ crossover under the experimental conditions, making the total crossover region broader. Second, we did not take into account the effect of dephasing in the low-voltage region, where we used the result for noninteracting electrons. It is easy to see from Eq. (39) that this will lead to a relative correction of order $V / V_{c} g$, which will provide a smooth matching with the strong-dephasing (high-voltage) regime (48).

We also note that the linear behavior (17) could not be accessed reliably in this experiment, since it requires, in view of the very small value of the numerical coefficient (18), very large voltages $V / V_{c} \gtrsim 1000$, while the dephasing was setting in at voltages several times smaller. A reliable observation of this linear behavior predicted by LK thus requires samples with still larger values of the dimensionless conductance $g$.

At high voltages, the experimentally observed decay of the conductance fluctuations is consistent with our prediction of the $1 / V$ decay, Eq. (48) (see Fig. 3). Indeed, the value $\gamma=1.28 \pm 0.12$ of the exponent of the power-law decay, $\left\langle\delta g^{2}\right\rangle \propto V^{-\gamma}$ is in good agreement with our result, $\gamma=1$. We thus disagree with the conclusion of the authors of Ref. 10 who argued, based on a naive estimate of the dephasing effect, that the electron-electron scattering is not sufficient to convert the LK enhancement mechanism into the observed fall-off, and ascribed this behavior to electron-phonon scattering. Experiments on samples with lower conductances might give more data in the strong-dephasing range and thus allow a more accurate experimental determination of the exponent of the power law.

\section{SUMMARY}

In this article, we investigated the amplitude of mesoscopic fluctuations of the differential conductance of a metallic wire at arbitrary bias voltage $V$. For noninteracting electrons, we have found, by using the Keldysh diagram technique, that the variance $\left\langle\delta g^{2}\right\rangle$ increases monotonically with $V$. The asymptotic large- $V$ behavior is $\left\langle\delta g^{2}\right\rangle \sim V / V_{c}$ (where $e V_{c}=D / L^{2}$ is the Thouless energy), in agreement with the prediction by Larkin and Khmelnitskii. We find, however, that this asymptotics has a very small numerical prefactor and sets in at very large $V / V_{c}$ only, so that it is very difficult to observe it reliably in the experiment. This highvoltage behavior is preceded by a crossover regime, $V / V_{c}$ $\$ 30$, where the conductance variance increases by a factor $\sim 3$ as compared to its value in the regime of universal conductance fluctuations (i.e., at $V \rightarrow 0$ ). We further analyze, in the framework of the path-integral technique, the effect of dephasing due to the electron-electron interaction on $\left\langle\delta g^{2}\right\rangle$ at high voltages. With the Coulomb interaction taken into account, the amplitude of conductance fluctuations becomes a nonmonotonic function of $V$. Specifically, $\left\langle\delta g^{2}\right\rangle$ shows a maximum at $V / V_{c} \sim g$ and drops as $1 / V$ for higher voltages. In this regime, the conductance fluctuations are dominated by quantum-coherent regions of the wire of a length

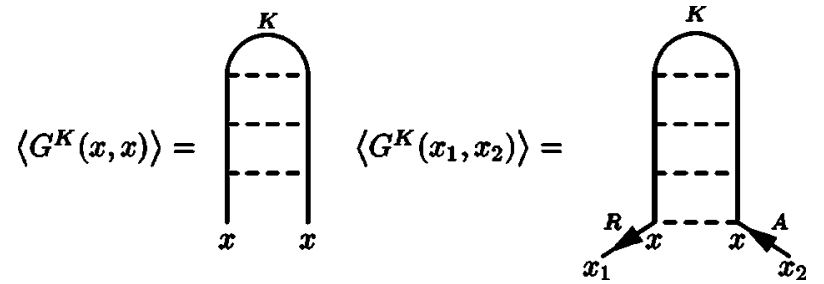

FIG. 4. Diagrammatic representation of the relation between the averaged Keldysh function $\left\langle G^{K}\left(x_{1}, x_{2}\right)\right\rangle$ and the averaged diagonal Keldysh function $\left\langle G^{K}(x, x)\right\rangle$, Eq. (A3), which is used in the derivation of Eq. (1).

$\sim L\left(g V_{c} / V\right)^{1 / 4}$ adjacent to the reservoirs. Our results are in good agreement with available experimental data.

\section{ACKNOWLEDGMENTS}

Useful discussions with I.V. Aleiner, B.L. Altshuler, C. Strunk, and C. Schönenberger are gratefully acknowledged. We also thank C. Strunk and C. Schönenberger for providing us with details of experimental data of Refs. 10 and 11. Part of this work was done when the authors participated at the Workshop "Quantum Transport and Correlations in Mesoscopic Systems and Quantum Hall Effect" at the Max-PlanckInstitut für Physik komplexer Systeme in Dresden.

\section{APPENDIX A: KELDYSH DIAGRAM FOR THE CURRENT}

Here we give the derivation of Eq. (1), which relates the current to the impurity averaged diagonal Keldysh function. We begin by expressing the averaged Keldysh function $\left\langle G^{K}\left(x_{1}, x_{2}\right)\right\rangle$ using the averaged diagonal Keldysh function $\left\langle G^{K}(x, x)\right\rangle$. The off-diagonal components of the kinetic equations for the matrix Green's function add up to

$$
(\epsilon-\hat{H}) G^{K}+G^{K}(\epsilon-\hat{H})=\frac{i}{\tau}\left[\left(2 n_{\epsilon}-1\right) G^{A}+G^{R}\left(2 n_{\epsilon}-1\right)\right]
$$

(here impurity averaging is implied). Using the gradient expansion, ${ }^{15}$ one finds

$$
\begin{aligned}
G^{K}(x, p)= & \frac{i}{\tau}\left\{\left[2 n_{\epsilon}(x)-1\right] G^{R}(p) G^{A}(p)+i \partial_{x} n_{\epsilon}(x)\right. \\
& \left.\times\left[G^{R}(p) \partial_{p} G^{A}(p)-G^{A}(p) \partial_{p} G^{R}(p)\right]\right\} .
\end{aligned}
$$

This is equivalent to the relation

$$
\left\langle G^{K}\left(x_{1}, x_{2}\right)\right\rangle=\frac{1}{2 \pi \nu \tau} \int d x\left\langle G^{R}\left(x_{1}, x\right)\right\rangle\left\langle G^{K}(x, x)\right\rangle\left\langle G^{A}\left(x, x_{2}\right)\right\rangle .
$$

This relation can be easily understood diagramatically (see Fig. 4). Using the relation ${ }^{15}$ between the function $G^{<} \equiv\left(G^{K}+G^{A}-G^{R}\right) / 2$ and the electron density matrix $\rho$,

$$
G^{<}\left(x_{1}, t ; x_{2}, t\right)=i N \rho\left(x_{1}, x_{2}, t\right)
$$

( $N$ is the particle number), one finds 


$$
I_{x}(x)=\left.\frac{(-e)}{m} \int \frac{d \epsilon}{2 \pi} \frac{\partial}{\partial x} G^{<}\left(x, x^{\prime}\right)\right|_{x^{\prime}=x} .
$$

The contribution from the term $G^{A}-G^{R}$ is proportional to the spectral density and does not depend on the state of the system. Therefore only $G^{K}$ contributes to the current, ${ }^{15}$

$$
\left\langle I_{x}(x)\right\rangle=\left.\frac{(-e)}{2 m} \frac{\partial}{\partial x} \int \frac{d \epsilon}{2 \pi}\left\langle G^{K}\left(x, x^{\prime}\right)\right\rangle\right|_{x^{\prime}=x} .
$$

Inserting Eq. (A3) into Eq. (A6) gives Eq. (1).

\section{APPENDIX B: EVALUATION OF THE KELDYSH DIAGRAMS}

In this appendix we calculate the diagrams that arise in the Keldysh technique and contribute to the correlation function of currents (see Sec. II). We will show that the sum of all the diagrams yields Eq. (2). We begin by presenting the expressions for the vertex factors (Hikami boxes). Because of their local character (the electron Green's function decays exponentially on the scale of the mean free path $l$, which is much smaller than the system size $L$ ), they can be calculated in the momentum space.

The right Hikami box (containing two Keldysh vertices) of the diagram a-diff is of second order in the momenta:

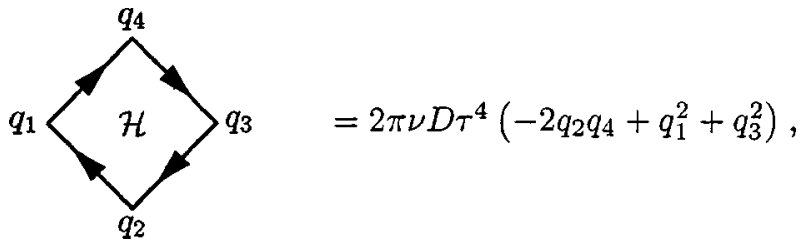

where the $q_{i}$ are the incoming momenta and $q_{1}+q_{2}+q_{3}+q_{4}$ $=0$. The left box (containing two velocity vertices) is of zeroth order:

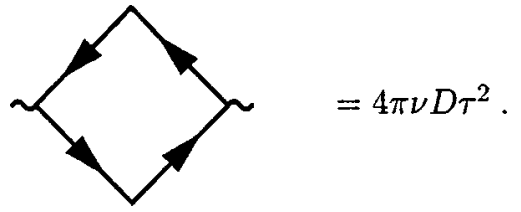

Here $D$ is the diffusion constant, $\nu$ is the density of states and $\tau^{-1}$ is the scattering rate characterizing the disorder strength. For the diagram b-diff the vertex factors are of first order in the momenta:

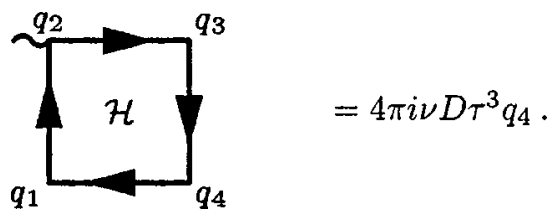

The vertex factors of a-coop and b-coop are the same as the vertex factors of b-diff up to extra signs arising from the direction of the Greens functions.

The vertex factors of the diagrams c-diff and c-coop are only needed to zeroth order. Since these diagrams contain only one diffuson/Cooperon, this gives a result of the same order in the momenta as the other diagrams (containing one more diffuson/Cooperon) evaluated up to second order in the momenta.

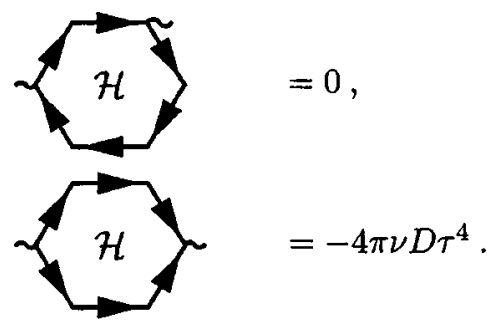

Using these vertex factors and changing to real space representation, the expressions corresponding to the diagrams are

$$
\begin{aligned}
\langle\delta I \delta I\rangle_{\mathrm{a}-\text { diff }}= & \left(\frac{e}{4 \pi i}\right)^{2}\left(\frac{1}{2 \pi \nu \tau}\right)^{2} 4 \pi \nu D \tau^{4} 2 \pi \nu D \tau^{2} \int d \epsilon_{1} d \epsilon_{2} \int \frac{d x_{1} d x_{2}}{L^{2}}\left[2 \frac{\partial}{\partial x_{1}} G_{\epsilon_{1}}^{K}\left(x_{1}\right) \frac{\partial}{\partial x_{2}} G_{\epsilon_{2}}^{K}\left(x_{2}\right) \mathcal{P}_{\epsilon_{1}-\epsilon_{2}} \mathcal{P}_{\epsilon_{2}-\epsilon_{1}}\right. \\
& \left.-\frac{\partial^{2}}{\partial x_{1}^{2}} \mathcal{P}_{\epsilon_{1}-\epsilon_{2}} \mathcal{P}_{\epsilon_{2}-\epsilon_{1}} G_{\epsilon_{1}}^{K}\left(x_{1}\right) G_{\epsilon_{2}}^{K}\left(x_{2}\right)-\mathcal{P}_{\epsilon_{1}-\epsilon_{2}} \frac{\partial^{2}}{\partial x_{2}^{2}} \mathcal{P}_{\epsilon_{2}-\epsilon_{1}} G_{\epsilon_{1}}^{K}\left(x_{1}\right) G_{\epsilon_{2}}^{K}\left(x_{2}\right)\right], \\
\langle\delta I \delta I\rangle_{\mathrm{b} \text {-diff }}= & \left(\frac{e}{4 \pi i}\right)^{2}\left(\frac{1}{2 \pi \nu \tau}\right)^{2}\left(4 \pi \nu D \tau^{3}\right)^{2} \int d \epsilon_{1} d \epsilon_{2} \int \frac{d x_{1} d x_{2}}{L^{2}}\left[\frac{\partial}{\partial x_{1}} \mathcal{P}_{\epsilon_{1}-\epsilon_{2}} \frac{\partial}{\partial x_{2}} \mathcal{P}_{\epsilon_{1}-\epsilon_{2}} G_{\epsilon_{1}}^{K}\left(x_{1}\right) G_{\epsilon_{2}}^{K}\left(x_{2}\right)+\mathrm{c} . \mathrm{c} .\right], \\
\langle\delta I \delta I\rangle_{\mathrm{a}-\text { coop }}= & \left(\frac{e}{4 \pi i}\right)^{2}\left(\frac{1}{2 \pi \nu \tau}\right)^{2}\left(4 \pi \nu D \tau^{3}\right)^{2} \int d \epsilon_{1} d \epsilon_{2} \int \frac{d x_{1} d x_{2}}{L^{2}} \mathcal{P}_{\epsilon_{1}-\epsilon_{2}} \mathcal{P}_{\epsilon_{2}-\epsilon_{1}} \frac{\partial}{\partial x_{1}} G_{\epsilon_{1}}^{K}\left(x_{1}\right) \frac{\partial}{\partial x_{2}} G_{\epsilon_{2}}^{K}\left(x_{2}\right), \\
\langle\delta I \delta I\rangle_{\mathrm{b}-\text { coop }}= & \left(\frac{e}{4 \pi i}\right)^{2}\left(\frac{1}{2 \pi \nu \tau}\right)^{2}\left(4 \pi \nu D \tau^{3}\right)^{2} \int d \epsilon_{1} d \epsilon_{2} \int \frac{d x_{1} d x_{2}}{L^{2}}\left(\frac{\partial^{2}}{\partial x_{1} \partial x_{2}} \mathcal{P}_{\epsilon_{1}-\epsilon_{2}}\right) \mathcal{P}_{\epsilon_{1}-\epsilon_{2}} G_{\epsilon_{1}}^{K}\left(x_{1}\right) G_{\epsilon_{2}}^{K}\left(x_{2}\right),
\end{aligned}
$$




$$
\langle\delta I \delta I\rangle_{\mathrm{c}-\text { coop }}=\left(\frac{e}{4 \pi i}\right)^{2}\left(\frac{1}{2 \pi \nu \tau}\right)^{2}\left(-4 \pi \nu D \tau^{4}\right) \int d \epsilon_{1} d \epsilon_{2} \int \frac{d x_{1} d x_{2}}{L^{2}} \delta\left(x_{1}-x_{2}\right) \mathcal{P}_{\epsilon_{1}-\epsilon_{2}} G_{\epsilon_{1}}^{K}\left(x_{1}\right) G_{\epsilon_{2}}^{K}\left(x_{2}\right),
$$

where $\mathcal{P}_{\epsilon_{1}-\epsilon_{2}}\left(x_{1}, x_{2}\right)$ is the diffusion propagator satisfying the equation

$$
\left\{D \frac{\partial^{2}}{\partial x_{1}^{2}}+i\left[\epsilon_{1}+\phi_{1}\left(x_{1}\right)-\epsilon_{2}-\phi_{2}\left(x_{1}\right)\right]\right\} \mathcal{P}_{\epsilon_{1}-\epsilon_{2}}\left(x_{1}, x_{2}\right)=-\left(2 \pi \nu \tau^{2}\right)^{-1} \delta\left(x_{1}-x_{2}\right),
$$

and $\phi_{1,2}$ are the electrostatic potentials corresponding to the voltages $V_{1,2}$. Using the identity

$$
\frac{1}{4} \frac{\partial^{2}}{\partial x_{1} \partial x_{2}} \mathcal{P}^{2}\left(x_{1}, x_{2}\right)=\left[\frac{\partial^{2}}{\partial x_{1} \partial x_{2}} \mathcal{P}\left(x_{1}, x_{2}\right)-\frac{1}{4 \pi \nu D \tau^{2}} \delta\left(x_{1}-x_{2}\right)\right] \mathcal{P}\left(x_{1}, x_{2}\right),
$$

we obtain

$$
\begin{gathered}
\langle\delta I \delta I\rangle_{\mathrm{a} \text {-diff } \mathrm{b}-\mathrm{diff}}=-\left(D \tau^{2}\right)^{2} \int d \epsilon_{1} d \epsilon_{2}\left[\left|\mathcal{P}_{\epsilon_{1}-\epsilon_{2}}\right|^{2}+\frac{1}{2} \operatorname{Re} \mathcal{P}_{\epsilon_{1}-\epsilon_{2}}^{2}\right] \frac{\partial}{\partial x_{1}} G^{K}\left(x_{1}\right) \frac{\partial}{\partial x_{2}} G^{K}\left(x_{2}\right), \\
\langle\delta I \delta I\rangle_{\mathrm{c}-\text { diff }}=0 \\
\langle\delta I \delta I\rangle_{\mathrm{a} \text {-coop }}=-\left(D \tau^{2}\right)^{2} \int d \epsilon_{1} d \epsilon_{2} \int \frac{d x_{1} d x_{2}}{L^{2}}\left|\mathcal{P}_{\epsilon_{1}-\epsilon_{2}}\right|^{2} \frac{\partial}{\partial x_{1}} G^{K}\left(x_{1}\right) \frac{\partial}{\partial x_{2}} G^{K}\left(x_{2}\right), \\
\langle\delta I \delta I\rangle_{\mathrm{b} \text {-coop+c-coop }}=-\left(D \tau^{2}\right)^{2} \int d \epsilon_{1} d \epsilon_{2} \int \frac{d x_{1} d x_{2}}{L^{2}} \frac{1}{2} \operatorname{Re} \mathcal{P}_{\epsilon_{1}-\epsilon_{2}}^{2} \frac{\partial}{\partial x_{1}} G^{K}\left(x_{1}\right) \frac{\partial}{\partial x_{2}} G^{K}\left(x_{2}\right) .
\end{gathered}
$$

Adding up these equations and rescaling the propagator by a factor $2 \pi \nu D \tau^{2}, \Pi=2 \pi \nu D \tau^{2} \mathcal{P}$, we obtain Eq. (2), which is written in units of $\left[e^{2} /(2 \pi)\right]^{2}=\left(e^{2} / h\right)^{2}$.

\section{APPENDIX C: CROSSOVER FROM THE LINEAR RESPONSE TO THE LARKIN-KHMELNITSKII REGIME}

In this appendix we calculate the variance $\left\langle\delta g^{2}\right\rangle$ as a function of the bias voltage in the full range from the UCF regime $\left(V / V_{c} \ll 1\right)$ to the LK asymptotic regime $\left(V / V_{c} \gg 1\right)$. In addition to the asymptotics equation (17), there are contributions to the variance of the conductance that do not grow asymptotically as $V / V_{c}$ but dominate in the intermediate regime. First of all, there is a constant contribution

$$
\langle\delta g \delta g\rangle_{0}=\frac{8}{15},
$$

which gives the familiar UCF result in the limit of zero bias voltage. Second, there is a contribution from the term $\langle\delta g \delta g\rangle_{1}$ containing one energy integration, which can be evaluated as

$$
\begin{aligned}
&\langle\delta g \delta g\rangle_{1}= 32 \int_{0}^{V / V_{c}} d z \frac{\partial}{\partial \alpha} \operatorname{Tr}\left[2\left|\Pi_{V / V_{c}-z}\right|^{2}+\operatorname{Re} \Pi_{V / V_{c}-z}^{2}\right]_{\alpha=0} \\
&= \frac{720+16\left(\frac{V}{V_{c}}\right)^{2}-360(-1)^{1 / 4} \sqrt{\frac{V}{V_{c}}}\left\{\cot \left[(-1)^{1 / 4} \sqrt{\frac{V}{V_{c}}}\right]+\operatorname{coth}\left[(-1)^{1 / 4} \sqrt{\frac{V}{V_{c}}}\right]\right\}}{45\left(\frac{V}{V_{c}}\right)^{2}} \\
&+\frac{8}{45}-16 \sum_{n=1}^{\infty} \frac{\left(\frac{V}{V_{c}}\right)^{2}-n^{4} \pi^{4}}{\left.n^{4} \pi^{4}+\left(\frac{V}{V_{c}}\right)^{2}\right]^{2}},
\end{aligned}
$$

where Eqs. (C2) and (C3) arise from the contributions of $|\Pi|^{2}$ and Re $\Pi^{2}$, respectively. In the limit $V / V_{c} \rightarrow \infty$, the sum of Eqs. (C2) and (C3) saturates at 8/15. Then there is the part of $\langle\delta g \delta g\rangle_{2}$ containing Re $\Pi^{2}$, which also does not contribute to the LK asymptotic behavior but gives a contribution which saturates towards a constant as the voltage is increased: 


$$
\begin{aligned}
\langle\delta g \delta g\rangle_{2, \operatorname{Re}} & =-\left.16 \int_{0}^{V_{1} / V_{c}} d z_{1} \int_{0}^{V_{2} / V_{c}} d z_{2} \frac{\partial^{2}}{\partial \alpha^{2}} \operatorname{Tr} \operatorname{Re} \Pi_{z_{1}-z_{2}}^{2}\right|_{\alpha=0}=32 \sum_{m, n>0} \frac{\left.2\left(\frac{V}{V_{c}}\right)^{2}\left[m^{4} \pi^{4}+m^{2} n^{2} \pi^{4}+n^{4} \pi^{4}+\left(\frac{V}{m^{2} n^{2} \pi^{4}}\right)^{2}\right] m^{4} \pi^{4}+\left(\frac{V}{V_{c}}\right)^{2}\right]\left[n^{4} \pi^{4}+\left(\frac{V}{V_{c}}\right)^{2}\right]}{(\langle n|y| m\rangle)^{2}}{ }^{\rightarrow 0.1905,}
\end{aligned}
$$

where again $|n\rangle=\sqrt{2} \sin (n \pi y)$. Finally, the $|\Pi|^{2}$ contribution to $\langle\delta g \delta g\rangle_{2}$ will also be modified at finite $V / V_{c}$ as compared to its asymptotics given by Eq. (17). The corresponding expression ${ }^{21}$ is too cumbersome, and we do not reproduce it here. Combining all the contributions we get the result shown graphically in Fig. 1.

\section{APPENDIX D: SUBLEADING TERMS IN THE STRONG-DEPHASING REGIME}

In this appendix we show that the terms $\langle\delta g \delta g\rangle_{0}$ and $\langle\delta g \delta g\rangle_{1}$ remain small compared to the leading term, $\langle\delta g \delta g\rangle_{2}$, at high voltages, $V / V_{c} \gg g$, i.e., in the regime of strong dephasing. Let us first analyze the behavior of the contribution (12),

$$
\langle\delta g \delta\rangle_{1}=\left.32 V_{c} \int_{0}^{V_{1} / V_{c}} d z_{1} \frac{\partial}{\partial V_{1}} \Xi_{z_{1}-V_{2} / V_{c}}\right|_{V_{1}=V_{2}=V}
$$

which to leading order in $V_{c} / V$ is

$$
\langle\delta g \delta\rangle_{1}=32\left[\left.\pi V_{c} \frac{\partial}{\partial V_{1}} \widetilde{\Xi}(0)\right|_{V_{1}=V_{2}}+\int d t \widetilde{\Xi}(t)\right] .
$$

The last term in the brackets is just twice $\langle\delta g \delta g\rangle_{0}$, which we will discuss separately below. The first term in the brackets gives up to a numerical prefactor

$$
\langle\delta g \delta g\rangle_{1} \sim\left(\frac{V_{c} g}{V}\right)^{5 / 4}
$$

Clearly, Eq. (D3) is not only smaller by a factor $1 / g$ compared to the leading term, Eq. (48), at the lower bound of the strong dephasing regime $V / V_{c} \sim g$, but also decays faster with increasing voltage.

It remains to show that the contribution of $\langle\delta g \delta g\rangle_{0}$ also is small compared to Eq. (48). Setting $V_{1}=V_{2}=V$ in Eq. (39), we get

$$
\begin{aligned}
\Xi_{0}= & 2 \int_{0}^{1} d y_{1} d y_{2} \int_{0}^{\infty} d \tau_{1} d \tau_{2} \int_{\xi_{1}(0)=y_{2}}^{\xi_{1}\left(\tau_{1}\right)=y_{1}} \mathcal{D} \xi_{1}\left(t_{1}\right) \int_{\xi_{2}(0)=y_{2}}^{\xi_{2}\left(\tau_{2}\right)=y_{1}} \mathcal{D} \xi_{2}\left(t_{2}\right) \\
& \times \exp \left\{-\int_{0}^{\tau_{1}} d t_{1} \frac{\dot{\xi}_{1}^{2}}{4}-\int_{0}^{\tau_{2}} d t_{2} \frac{\dot{\xi}_{2}^{2}}{4}-\frac{2 V}{V_{c} g} F\left(y_{2}\right)\left|\tau_{1}-\tau_{2}\right|-\frac{2 V}{V_{c} g} \int_{0}^{\min \left(\tau_{1}, \tau_{2}\right)} d t\left|\xi_{1}-\xi_{2}\right| y_{2}\left(1-y_{2}\right)\right\} .
\end{aligned}
$$

Since the bare field fluctuations in the time interval between $\tau_{1}$ and $\tau_{2}$ strongly suppress interference, relevant contributions are given by paths with $\tau_{1}$ and $\tau_{2}$ close to each other. This allows us to approximate the path integral by two trajectories propagating for the same time, keeping only the plain exponential suppression factor to account for the time difference:

$$
\Xi_{0}=2 \sqrt{2} \int_{0}^{1} d y_{1} d y_{2} \int_{0}^{\infty} d \tau_{1} \int_{0}^{\tau_{1}} d \tau_{2} \exp \left\{-\frac{2 V}{V_{c} g} F\left(y_{2}\right)\left|\tau_{1}-\tau_{2}\right|\right\} \int_{\xi(0)=0}^{\xi\left(\tau_{1}\right)=0} \mathcal{D} \xi\left(t_{1}\right) \exp \left\{-\int_{0}^{\tau_{1}} d t\left[\frac{\dot{\xi}^{2}}{4}+\frac{2 V}{V_{c} g} \sqrt{2}|\xi| y_{2}\left(1-y_{2}\right)\right]\right\},
$$

where again we transformed to the sum and the difference of the coordinates $\xi_{1}, \xi_{2}$ and carried out the trivial path integral over the sum. We can also perform the integral over $\tau_{2}$, 


$$
\begin{aligned}
& \Xi_{0}=\sqrt{2} \frac{V_{c} g}{V} \int_{0}^{1} \frac{d y}{F(y)} \int_{0}^{\infty} d \tau\left(1-e^{-\left(2 V / V_{c} g\right) F(y) \tau}\right) \int_{\xi(0)=0}^{\xi(\tau)=0} \mathcal{D} \xi(t) \exp \left\{-\int_{0}^{\tau} d t\left[\frac{\dot{\xi}^{2}}{4}+\frac{2 V}{V_{c} g} \sqrt{2}|\xi| y(1-y)\right]\right\} \\
&=\sqrt{2} \frac{V_{c} g}{V} \int_{0}^{1} \frac{d y}{F(y)} \int_{0}^{\infty}\left[\frac{d \tau}{\left[\frac{2 V}{V_{c} g} \sqrt{2} y(1-y)\right]^{1 / 3}}\left(1-\exp \left\{-\frac{\frac{2 V}{V_{c} g} F(y) \tau}{\left[\frac{2 V}{V_{c} g} \sqrt{2} y(1-y)\right]^{2 / 3}}\right\}\right)\right. \\
& \mathcal{I}(0, \tau),
\end{aligned}
$$

where $\left(\partial_{\tau}-\partial_{\eta}^{2}+|\eta|\right) \mathcal{I}(\eta, \tau)=\delta(\eta) \delta(\tau)$. This integral is dominated by $y$ close to 0 or 1 , and should be cut off at $y \sim y_{c}$ and $(1$ $-y) \sim y_{c}$, where $y_{c}$ is given by Eq. (47). This yields

$$
\langle\delta g \delta g\rangle_{0} \sim g \frac{V_{c}}{V},
$$

so that $\langle\delta g \delta g\rangle_{0}$ remains smaller by a factor $\sim 1 / g$ than $\langle\delta g \delta g\rangle_{2}$ in the strong dephasing regime. Since both Eqs. (D3) and (D7) are small compared to Eq. (48) at high voltages, we thus have shown that the asymptotic suppression of the conductance fluctuations is given by Eq. (48).

*Also at Petersburg Nuclear Physics Institute, 188350 St. Petersburg, Russia.

${ }^{1}$ B. L. Altshuler, Pis'ma Zh. Eksp. Teor. Fiz. 41, 530 (1985) [JETP Lett. 41, 648 (1985)].

${ }^{2}$ P. A. Lee and A. D. Stone, Phys. Rev. Lett. 55, 1622 (1985).

${ }^{3}$ P. A. Lee, A. D. Stone, and H. Fukuyama, Phys. Rev. B 35, 1039 (1987).

${ }^{4}$ A. I. Larkin and D. E. Khmel'nitskii, Zh. Eksp. Teor. Fiz. 91, 1815 (1986) [Sov. Phys. JETP 64, 1075 (1986)]; Phys. Scr., T T14, 4 (1986).

${ }^{5}$ R. A. Webb, S. Washburn, and C. P. Umbach, Phys. Rev. B 37, 8455 (1988).

${ }^{6}$ S. B. Kaplan, Phys. Rev. B 38, 7558 (1988).

${ }^{7}$ D. C. Ralph, K. S. Ralls, and R. A. Buhrman, Phys. Rev. Lett. 70, 986 (1993).

${ }^{8}$ R. Schäfer, K. Hecker, and H. Hegger, Phys. Rev. B 53, 15964 (1996).

${ }^{9}$ R. Häussler, H. B. Weber, and H. v. Löhneysen, J. Low Temp. Phys. 118, 467 (2000).

${ }^{10}$ C. Terrier, D. Babić, C. Strunk, T. Nussbaumer, and C. Schönenberger, Europhys. Lett. 59, 437 (2002) and (private communi- cation). Experimental data discussed but not explicitly presented in this paper can be found in Ref. 11.

${ }^{11}$ C. Terrier, Ph.D. thesis, Universität Basel, 2002.

${ }^{12}$ B. L. Altshuler and A. G. Aronov, in Electron-Electron Interaction in Disordered Conductors, edited by A. L. Efros and M. Pollak (Elsevier, Amsterdam, 1985).

${ }^{13}$ B. L. Altshuler, A. G. Aronov, and D. E. Khmelnitsky, J. Phys. C 15, 7367 (1982).

${ }^{14}$ L. V. Keldysh, Zh. Eksp. Teor. Fiz. 47, 1515 (1964) [Sov. Phys. JETP 20, 1018 (1965)].

${ }^{15}$ J. Rammer and H. Smith, Rev. Mod. Phys. 58, 323 (1986).

${ }^{16}$ Note that the diagrams c-diff and c-coop, as well as some possibilities of inserting additional impurity lines in the Hikami boxes, are missing in Fig. 1 of Ref. 4. However, the expression given in Ref. 4 appears to give correctly the sum of all diagrams.

${ }^{17}$ Sh. Kogan, Electronic Noise and Fluctuations in Solids (Cambridge University Press, Cambridge, 1996).

${ }^{18}$ K. E. Nagaev, Phys. Rev. B 57, 4628 (1998).

${ }^{19}$ For a review, see Ya. M. Blanter and M. Büttiker, Phys. Rep. 336, 1 (2000).

${ }^{20}$ I. L. Aleiner and Ya. M. Blanter, Phys. Rev. B 65, 115317 (2002).

${ }^{21}$ T. Ludwig, diploma thesis, Universität Karlsruhe, 2002. 